\title{
Intracranial EEG Reveals a Time- and Frequency-Specific Role for the Right Inferior Frontal Gyrus and Primary Motor Cortex in Stopping Initiated Responses
}

\author{
Nicole Swann, ${ }^{1,2}$ Nitin Tandon, ${ }^{3}$ Ryan Canolty, ${ }^{4}$ Timothy M. Ellmore, ${ }^{3}$ Linda K. McEvoy, ${ }^{5}$ Stephen Dreyer, ${ }^{3}$ \\ Michael DiSano, ${ }^{3}$ and Adam R. Aron ${ }^{1,2}$ \\ ${ }^{1}$ Neuroscience Program, University of California, San Diego, La Jolla, California 92093-0662, 22Department of Psychology, University of California, San \\ Diego, La Jolla, California 92093-0109, ${ }^{3}$ Department of Neurosurgery, University of Texas Medical School at Houston, Houston, Texas 77030, ${ }^{4}$ Helen Wills \\ Neuroscience Institute, University of California, Berkeley, Berkeley, California 94720-3192 and Department of Electrical Engineering and Computer \\ Sciences, University of California, Berkeley, California 94720-1770, and ${ }^{5}$ Department of Radiology, University of California, San Diego, San Diego, \\ California 92103-8756
}

\begin{abstract}
Inappropriate response tendencies may be stopped via a specific fronto/basal ganglia/primary motor cortical network. We sought to characterize the functional role of two regions in this putative stopping network, the right inferior frontal gyrus (IFG) and the primary motor cortex (M1), using electocorticography from subdural electrodes in four patients while they performed a stop-signal task. On each trial, a motor response was initiated, and on a minority of trials a stop signal instructed the patient to try to stop the response. For each patient, there was a greater right IFG response in the beta frequency band $(\sim 16 \mathrm{~Hz})$ for successful versus unsuccessful stop trials. This finding adds to evidence for a functional network for stopping because changes in beta frequency activity have also been observed in the basal ganglia in association with behavioral stopping. In addition, the right IFG response occurred $100-250 \mathrm{~ms}$ after the stop signal, a time range consistent with a putative inhibitory control process rather than with stop-signal processing or feedback regarding success. A downstream target of inhibitory control is M1. In each patient, there was alpha/beta band desynchronization in M1 for stop trials. However, the degree of desynchronization in M1 was less for successfully than unsuccessfully stopped trials. This reduced desynchronization on successful stop trials could relate to increased GABA inhibition in M1. Together with other findings, the results suggest that behavioral stopping is implemented via synchronized activity in the beta frequency band in a right IFG/basal ganglia network, with downstream effects on M1.
\end{abstract}

\section{Introduction}

Cognitive control is required to stop inappropriate action. One way to study this experimentally is with the stop-signal task (Logan, 1994; Verbruggen and Logan, 2009). On each trial, the participant initiates a motor response and on a minority of trials tries to stop the response when a stop signal occurs. Behaviorally, such stopping is operationalized as the time to stop the response: stopsignal reaction time (SSRT). Loss-of-function studies show that the dorsomedial frontal cortex and the right inferior frontal gyrus (IFG) are critical regions for stopping (Aron et al., 2003; Chambers et al., 2006, 2007; Floden and Stuss, 2006; Chen et al., 2008), and functional MRI affirms this (Rubia et al., 2003; Aron and Poldrack, 2006; Li et al., 2006; Aron et al., 2007; Chevrier et al., 2007; Chamberlain et al., 2008). Yet the precise functional role of these regions in behavioral stopping is still unclear.

Received July 13, 2009; revised Aug. 14, 2009; accepted Aug. 25, 2009.

N.S. is supported by a National Science Foundation graduate student fellowship. A.R.A. is supported by an Alfred P. Sloan Fellowship and National Institutes of Health (NIH) Grant 1R01DA026452-01A1. N.T. is supported by a K12 award from the NIH-funded Center for Clinical and Translational Sciences, UL1RR024148. We thank John Serences for helpful comments.

Correspondence should be addressed to Adam R. Aron, Department of Psychology, University of California, San Diego, 9500 Gilman Drive, La Jolla, CA 92093-0109. E-mail: adamaron@ucsd.edu.

DOI:10.1523/JNEUROSCI.3359-09.2009

Copyright $\odot 2009$ Society for Neuroscience $\quad$ 0270-6474/09/2912675-11\$15.00/0
While the right IFG may implement an inhibitory control function, it may also be important for stop-signal detection, working memory, response selection, and stimulus-driven attention (Corbetta and Shulman, 2002; Hampshire et al., 2007; Mars et al., 2008; Mostofsky and Simmonds, 2008; Zandbelt et al., 2008). One way to dissociate these functions is with the high temporal resolution of electrocencephalography (EEG). Studies with scalp EEG show that successfully stopped trials are associated with a larger negativity at $\sim 200 \mathrm{~ms}$ (after the stop signal) than unsuccessful stop trials or trials with no stop signal (Kok et al., 2004; Schmajuk et al., 2006; Liotti et al., 2007; Dimoska and Johnstone, 2008). This timing is consistent with a putative inhibitory function generated after the stop signal but before SSRT typically elapses (typically $\sim 250 \mathrm{~ms}$ ). However, as the spatial resolution of scalp EEG is limited, it is uncertain what is the neural source of this event-related potential.

We sought to elucidate the functional role of the right IFG by harnessing the high spatiotemporal resolution of electrocorticography (ECoG). We recorded directly from the cortical surface of patients undergoing presurgical monitoring. For each patient we selected a right IFG electrode in an unbiased manner and analyzed the ECoG for stop trials. If the right IFG implements an inhibitory control function, then we predicted it would show a 


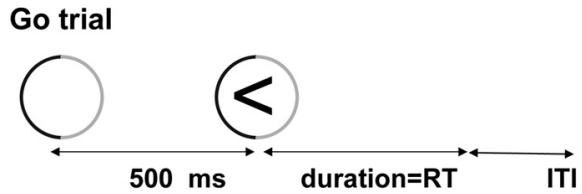

Stop trial

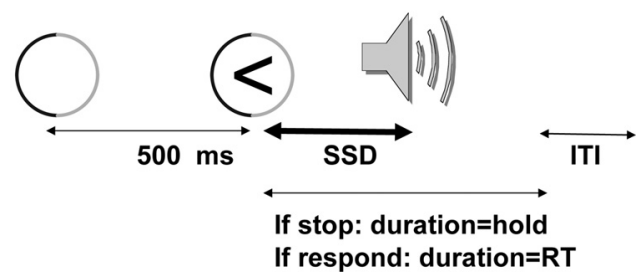

Figure 1. The conditional stop-signal task used in the electrocorticography experiment. Each trial begins with a cue circle (with, for example, the left half red, shown here as black, and the right half green, shown here as gray). The cue reminds the participant that the "critical" direction (red) is the left response and the "noncritical" direction (green) is the right response. The rules stay the same for the entire session. $0 n$ go trials, the participant has $1 \mathrm{~s}$ (the hold period) to press the left or right button in response to a stimulus. On a stop trial, a tone is played at some delay (SSD) after the arrow stimulus. The SSD changes dynamically throughout the experiment (increasing or decreasing by 50 ms depending on whether the participant stopped or not on prior stop trials). If the arrow stimulus is in the critical direction and a tone occurs, then the subject must try to stop, but if the arrow is in the noncritical direction and a tone occurs then the subject must respond anyway.

strong response within a time window ranging from the stop signal to the SSRT. Moreover, the frequency band of the response was also of interest. Finding a stopping-related response in right IFG within a particular frequency band might help to characterize the information-processing function of the right IFG within the wider putative frontobasal ganglia network. It has been hypothesized that communication between functionally/structurally connected brain regions may occur in specific frequency bands (Fries, 2005).

We also examined $\mathrm{M} 1$ - a downstream target of inhibitory control. Studies with transcranial magnetic stimulation (TMS) suggest that successful stop trials are associated with increased GABA-mediated inhibition in M1 (Sohn et al., 2002; Coxon et al., 2006; van den Wildenberg et al., 2009) and an fMRI study showed different responses for successful and unsuccessful stop trials in this region (Aron and Poldrack, 2006). ECoG could provide convergent evidence for understanding the dynamics of M1 during behavioral stopping in terms of the timing of the neural response and the frequency band.

\section{Materials and Methods}

Participants. Four right-handed patients (three female, mean age 32, range $20-47$ ) with medically refractory epilepsy were studied with ECoG. All patients had subdural electrode arrays implanted over their right hemispheres. Two of the patients (TA341 and TA344) were also studied with functional MRI on a similar task (before grid placement). One patient (TA344) had a right anterior temporal lobectomy 10 years before this study. Another patient (TS007) had a prior bilateral depth electrode evaluation and a past history of meningitis. All patients provided written informed consent according to Institutional Review Board guidelines at the University of Texas Medical School at Houston, Houston, TX.

The stop-signal task used for ECoG. The task was highly similar to that used in an earlier study [see the study by Aron et al. (2007) for full details]. Here it is reprised in brief, with key additional information. On each trial a left- or right-pointing arrow stimulus was presented (Fig. 1). This was displayed on a laptop computer screen placed on a table over the patient's bed in the epilepsy monitoring unit. Once the arrow stimulus was presented, the patient responded as fast as possible with a left or right key press (using index and middle fingers of either the left or right hand) (Table 1). [Note that hand use varied due to clinical exigencies, such as placement of intravenous lines. Prior research shows that the righthemisphere basis of stop-signal response inhibition is unlikely related to the hand used (Konishi et al., 1999; Chambers et al., 2006), and our results are consistent with this.] On a minority of trials (33\%), an auditory stop signal $(500 \mathrm{~Hz} ; 400 \mathrm{~ms}$ duration) was played at a particular stop-signal delay (SSD) subsequent to the arrow stimulus. When the patient detected the tone, he or she was required to try to stop the initiated response, but only if the arrow was pointing in a "critical" direction: leftward pointing for TS007 (both days of testing) and TS005, and rightward pointing for TA341 and TA344. [We note that with the exception of one auxiliary analysis (supplemental Fig. 3, available at www.jneurosci. org as supplemental material), the data presented here focus only on trials in which the arrow pointed in the critical direction. Thus, in what follows go trials are go critical, successful stop trials are successful stop critical, and unsuccessful stop trials are unsuccessful stop critical.]

In each block there were 32 stop and 64 go trials ( 96 trials total). In every 24 trials, there were 8 stop trials and 16 go trials, trial order was random, and the numbers of leftward- and rightward-pointing arrows were equal. Each trial began with a colored circular fixation ring (subtending 4.3 degrees of visual angle) appearing in the center of a white background screen. Half the circle was red (e.g., left side) and the other half green to help remind the patient of the critical direction. This was a constant arrangement for each patient's session. After $500 \mathrm{~ms}$, a black arrow (subtending 1.9 degrees of visual angle) was displayed within the fixation ring. The fixation ring and arrow remained on the screen for up to $1 \mathrm{~s}$ (limited hold), after which they disappeared, and the background screen was shown for an intertrial interval (ITI) of $1.4 \mathrm{~s}$. When the patient responded within the limited hold window, the fixation ring and arrow disappeared, and the background was shown for the remainder of the limited hold and ITI. A stop trial was identical to a go trial, except that a tone was played at some stop-signal delay after the arrow stimulus. If the response was stopped, then the arrow and fixation ring remained onscreen for the duration of the limited hold. If the subject responded, then the arrow and fixation ring disappeared, and the background screen was displayed for the remainder of the time. The SSD changed dynamically throughout the experiment, depending on the subject's behavior with respect to the critical key, to yield $\sim 50 \% p_{\text {stop }}$ [see the study by Aron et al. (2007) for precise details)]. Tracking in this way is desirable to achieve approximately equal numbers of successful and unsuccessful stop trials for ECoG analysis.

Before the experiment started, patients were trained to perform go and stop trials. Instructions made it clear that stopping and going were equally important and that it would not always be possible to stop. Each block was preceded by an instruction screen and was followed by feedback in the form of median-correct reaction time (RT), the number of discrimination errors on go trials, and the number of times when the patient incorrectly stopped when the arrow pointed in the noncritical direction (where going is always appropriate). During the course of each block, pauses were inserted every 24 trials to allow the patient to rest. We aimed to collect eight blocks ( 96 trials each) for each patient/session. However, fatigue made this impossible in some cases (Table 1).

The stop-signal task used for fMRI. The task/procedure used was identical to that reported in a prior fMRI study (Aron and Poldrack, 2006). Two patients (TA341 and TA344) performed three blocks/scans (6 min each) of a standard stop-signal task within the MRI magnet using their left hand to respond. The fMRI task was administered before subdural array placement and ECoG. Although the standard stop-signal task is slightly different from the conditional stop-signal task used for ECoG, both have been shown to activate the right IFG similarly (Aron and Poldrack, 2006; Aron et al., 2007). For fMRI, we used the standard task as a right IFG "localizer" because it enabled us to attain more stop trials per unit time.

Behavioral analysis. For both ECoG and fMRI sessions we computed median correct go RT, the percentage of successful stop trials, the mean stop-signal delay, the percentage of discrimination errors on go trials, and the percentage of response omissions on go trials. In most patients 
Table 1. Data collection parameters

\begin{tabular}{|c|c|c|c|c|c|}
\hline Patient & $\begin{array}{l}\text { Sampling } \\
\text { rate }(\mathrm{Hz})\end{array}$ & $\begin{array}{l}\text { Blocks } \\
\text { run }\end{array}$ & $\begin{array}{l}\text { Hand } \\
\text { used }\end{array}$ & $\begin{array}{l}\text { No. successful stop trials } \\
\text { (before artifact reject/after) }\end{array}$ & $\begin{array}{l}\text { No. unsuccessful stop trials } \\
\text { (before artifact reject/after) }\end{array}$ \\
\hline TS007 (day 1) & 200 & 8 & Left & $85 / 65$ & $43 / 32$ \\
\hline TSO07 (day 2) & 1000 & 8 & Right & $81 / 69$ & $47 / 41$ \\
\hline TSO05 & 500 & 4.5 & Right & $36 / 24$ & $44 / 26$ \\
\hline TA341 & 1000 & 7 & Left & $64 / 59$ & $58 / 49$ \\
\hline TA344 & 1000 & 8 & Left & $36 / 24$ & $92 / 64$ \\
\hline
\end{tabular}
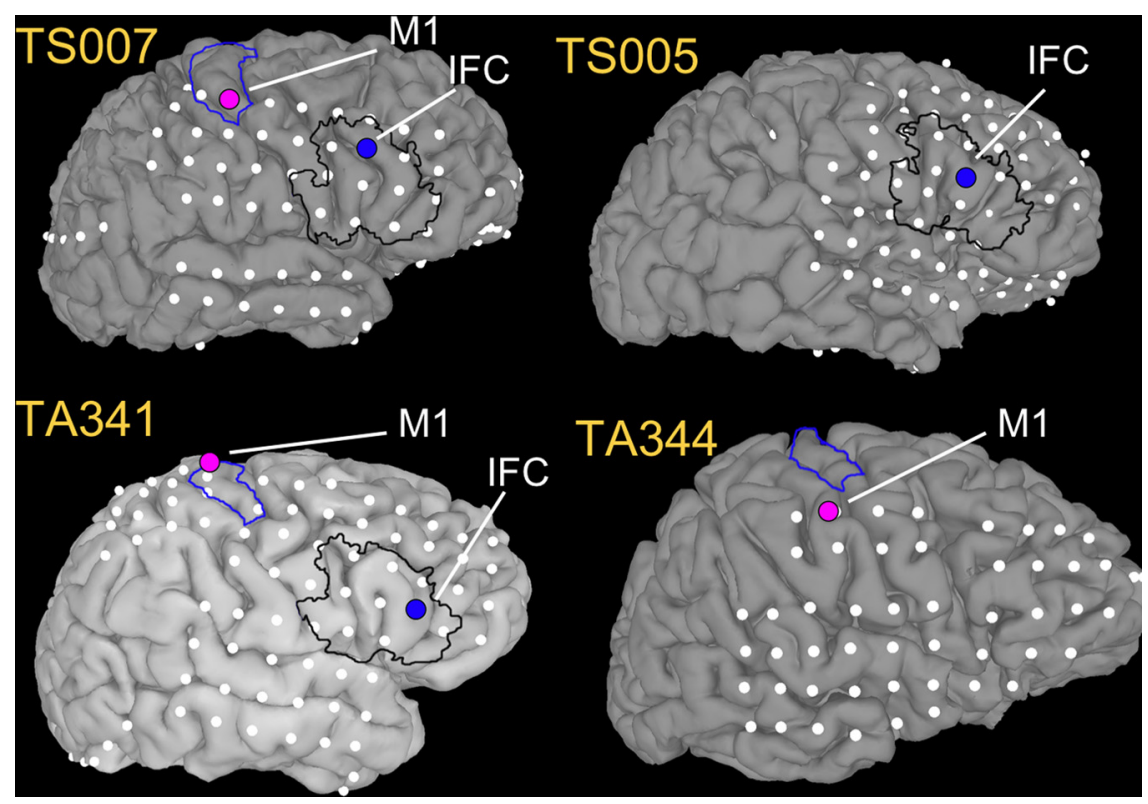

Figure 2. Location of intracranial grids on the right hemisphere. For each patient the ECOG grid is registered to the patient's own structural MRI. For the patients for whom we report a right IFG response, the probabilistically determined right IFG region is outlined in black (Eickhoff et al., 2005). For the patients for whom we report M1 results, the hand area of M1, determined by a neurosurgical expert, is outlined in blue. IFC, IFG cortex.

the dynamic tracking of SSD allowed convergence to $\sim 50 \%$ successful stop trials; however, this was not the case for all patients and we therefore estimated SSRT for everyone using the integration method (Verbruggen and Logan, 2009).

ECoG acquisition and filtering. Data were collected using an EEG 1100 Neurofax clinical Nihon Koden acquisition system. The number and placement of electrodes and the sampling rate were determined by clinical considerations (Table 1). Trial onsets for the behavioral task were sent to the ECoG data acquisition computer via transistor-transistor logic pulses. All data were referenced to a subdural electrode outside the regions of interest and then subsequently digitally re-referenced to a common average reference for analysis.

Electrode localizations. Subdural electrodes were localized through a procedure that involved registration to preoperative structural MRI (pre-MRI) and postoperative computed tomography (CT) scan. The pre-MRI was a high-resolution T1-weighted anatomical scan acquired on a $3 \mathrm{~T}$ Philips scanner. From this, a cortical surface model was generated (Dale et al., 1999). Following subdural electrode implantation, a high-resolution contiguous thin-slice CT was acquired (post-CT). The post-CT was coregistered to the pre-MRI with a rigidbody transformation algorithm using a mutual information cost function (Cox, 1996). The center of individual electrodes was localized using the post-CT. The subdural electrodes are visualized with FreeSurfer tools as spherical geometric objects (Fig. 2). To account for the possibility that nonlinear deformation of the cortical surface occurs after electrode implantation (Studholme et al., 2001), we modified the pial cortical surface model to disregard fissures and sulci, creating a smoothed envelope exactly fitting the shape and size of the subject's brain. The electrodes were then realigned to this new surface by placing them along a surface normal to the point on the envelope closest to their location on the deformed data from the time domain into the frequency domain. The transformed signal was then multiplied by many overlapping Gaussians centered at selected frequencies of interest and with different SDs depending on the center frequency. These values, and the number of Gaussians used, varied for different patients, according to their sampling rates. In all cases the lowest center frequency was $2.5 \mathrm{~Hz}$ and the highest was less than half the sampling rate (Nyquist cutoff), and always $<300 \mathrm{~Hz}$, the maximum high-frequency cutoff of the hardware. (Note that for TA341, for whom higher frequencies were contaminated by electrical noise, the maximum frequency examined was $30 \mathrm{~Hz}$.) (Note that this process is analogous to a Gabor wavelet analysis in the temporal domain but is performed in the frequency domain for computational efficiency.) The data were then separated into different groups, with each group having a different center frequency. An inverse fast Fourier transform was applied to data in each group, converting it back into the time domain. The resulting data, referred to as the analytic signal, had an equal number of time points as the original raw signal, but the signal is now separated into the different frequencies of interest. The amplitude of this analytic signal was used to calculate power, which is the dependent variable for the ECoG analysis. Next, the data were broken up with respect to events of interest (for instance the period of time around each stop signal), baseline corrected to account for prestimulus activity, and averaged (e.g., mean power was attained for all successful stop trials, across many frequencies, and for a specific period of time around the stop signal). Finally, the analytic power was converted to $z$ scores.

Taking the example of a single channel in the right IFG: the signal was filtered into many separate frequencies and the analytic signal was obtained as described above. For a single center frequency, and for each successful stop trial, data were extracted corresponding to the analytic signal from $1750 \mathrm{~ms}$ before the stop signal to $1750 \mathrm{~ms}$ after the stop signal ( -1750 to 1750 , where 

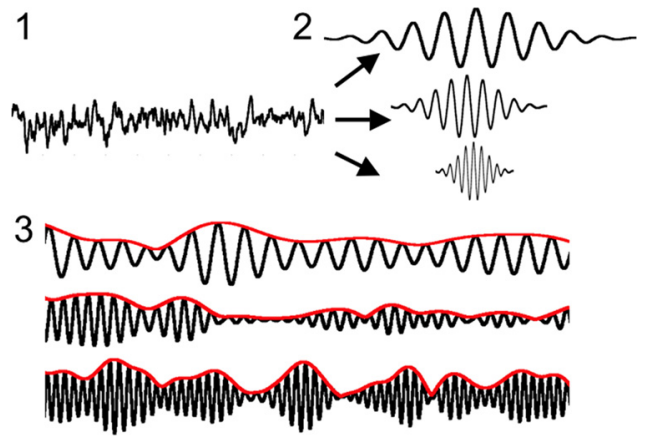

4

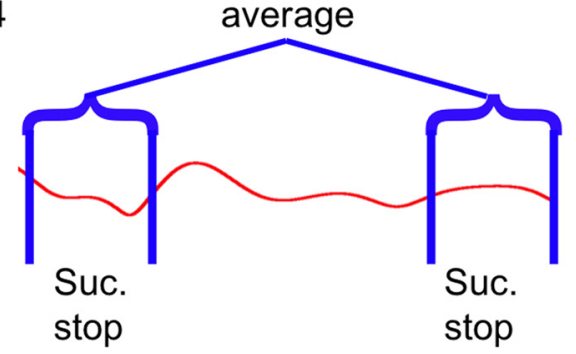

Figure 3. ECOG analysis procedure. (1) Raw EEG signal (after referencing to common average). (2) Filtering. The raw signal is filtered using a gabor wavelet technique into many separate frequencies (only 3 are shown for visualization purposes). (3) Deriving the analytic signal. Traces of the filtered signal for three representative frequencies are shown in black. The red line shows the analytic amplitude ("power") for each of these frequencies. It is this analytic value over time for each frequency that is used for the remainder of the analysis. (4) Epoching. All events of one trial type, e.g., successful (Suc.) stop, are grouped together. The analytic signals across the length of the trial are averaged together at each particular frequency. The panel shows one frequency band for illustration.

zero is the time at which the stop signal occurred). An average event-related time-frequency (ERTF) value was computed by averaging the absolute value of the analytic signal (power), across all such (successful stop) trials, producing a value that corresponded to the average power for each time point. An average prestimulus baseline was obtained by averaging power values, across time, for $500 \mathrm{~ms}$ of the intertrial interval $(-1750$ to $-1250 \mathrm{~ms}$ relative to the stop signal). This value was then subtracted from the ERTF values. This procedure was repeated for all filtered frequencies. The entire process described thus far was also performed for unsuccessful stop trials. A similar procedure, but with $0 \mathrm{~ms}$ corresponding to the go stimulus instead of the stop stimulus, was performed for M1 electrodes.

ECoG analysis: generating condition-specific z scores. Statistical inference used a published method (Canolty et al., 2007). This method uses bootstrapping to implement 10,000 runs time locked to random events to derive an ensemble of average analytic signal values for each separate frequency. Each generated number was used to offset from the real event indices, to generate data which was time locked to a random event, but was otherwise sampled in a manner analogous to the real data (same number of trials, same relative spacing). From each of these 10,000 runs a value corresponding to the amplitude of the analytic signal was stored. This ensemble was fit to a normal distribution, and an SD was derived representing the variability of the data at a particular frequency.

A $z$ score was obtained for each condition by dividing the ERTF values (see the prior section) for each frequency and time point by the abovederived SD for each frequency. This $z$ score was used to determine the probability that each deviation could occur by chance (uncorrected, two tailed). The procedure of obtaining $z$ scores was also performed for the difference between successful stopping and unsuccessful stopping conditions. In this case, ERTF values were obtained from the subtraction of the analytic signals for the two conditions, without baseline correction.

A correction for multiple comparisons was made using the falsediscovery rate (FDR) method. For all 10 or 11 electrodes in each patient's right IFG region (probabilistically determined from an anatomical atlas; Eickhoff et al., 2005) we calculated the $p$ values associated with the abovedetermined $z$ scores for each condition (successful and unsuccessful stop trials) across all time points of interest $(0-800 \mathrm{~ms}$ after the stop signal for the right IFG and 0-1250 ms after the go signal for M1) and across all frequencies. The $p$ values associated with all these $z$ scores were then sorted in ascending order $\left(p_{1}, p_{2}, p_{3}, \ldots p_{\mathrm{K}}, \ldots p_{\mathrm{M}}\right)$, and a threshold was determined according to the following:

$$
p_{K}>\left(K^{\star} \alpha\right) / M,
$$

where $p_{\mathrm{K}}$ is the FDR-corrected $\alpha$ value ( $p<0.01$ ), $K$ is the rank ordering of the $p$ value which corresponds to this threshold, $\alpha$ is 0.01 , and $M$ is the total number of comparisons.

ECoG analysis: between-conditions statistical comparison. In Results, we describe how a single right IFG and M1 electrode is selected for each patient for further analysis. Once this selection was performed, we tested the difference between successful and unsuccessful stop trials at this electrode in two ways. The first used the bootstrapping method above (by which all results were significant, $p<0.05$, but not FDR corrected). The second method used a "masking approach" which honed in on a particular time window and frequency range of interest (rather than all possible time and frequency ranges). For the masking approach, we selected the time and frequency range in a way that should not create bias to find a difference between conditions. For each patient, for the right IFG (or M1) electrode of interest, time-frequency activity was computed for all stop trials (compared to baseline) for the period ranging from the stop signal to $1 \mathrm{~s}$ later. The result was corrected for multiple comparisons using FDR $(p<0.01)$. This analysis, similar to that shown below (see Fig. 5 ), reveals a strong response in the beta band in all patients. A "mask" was derived for each patient which contained the data points in the beta band $(13-18 \mathrm{~Hz})$ from 0 to $1 \mathrm{~s}$ after the stop signal which surpassed the FDRcorrected threshold for all stop trials compared to the baseline. This mask was then applied to a time-frequency matrix of $z$ scores of the difference between successful and unsuccessful stop trials. For each data point within the mask, we noted (1) the direction of the difference and (2) how many of these data points ("observed" values) were statistically significant $(p<0.05)$ using the uncorrected, two-tailed method for obtaining $z$ scores for successful versus unsuccessful trials. The number of observed data points was then compared with the number of data points expected to be significant by chance alone (i.e., the total number of data points examined, $5 \%$ of the total number of data points examined). A $\chi^{2}$ test compared the disparity between the number of observed and expected observations for each patient.

fMRI acquisition and analysis. Data acquisition and analysis were the same as reported in a prior study (Aron and Poldrack, 2006) with two exceptions: (1) this was a Phillips 3T Scanner at the University of Texas Medical Center, Houston, and (2) functional images had the following parameter: 33 axial slices ( $3 \mathrm{~mm}$ slice thickness, 2.75 in-plan resolution) were sampled (TE $30 \mathrm{~ms}$, TR $2000 \mathrm{~ms}$, flip angle, 90 degrees). Preprocessing was done with tools from the FMRIB software library (www.fmrib. ox.ac.uk/fsl) as previously described (Aron and Poldrack, 2006). Model fitting was done for each patient separately and included the following events after convolution with a canonical hemodynamic response function: go, successful stop, unsuccessful stop, and nuisance events consisting of go trials on which subjects did not respond or made errors. Events were modeled at the time of the arrow stimulus. Temporal derivatives were included as covariates of no interest to improve statistical sensitivity. Null events were not explicitly modeled and therefore constituted an implicit baseline. For each scan, the contrasts go-null and successful stop-go were computed. Earlier we had shown that the contrast successful- unsuccessful stop is not effective at isolating frontal stopping-related activation (Aron and Poldrack, 2006; Aron et al., 2007; Xue et al., 2008; but see Rubia et al., 2003; Li et al., 2006). A higher-level fixed-effects analysis was performed across the three scans for each patient. Activations were corrected for multiple comparisons at $z>2.0, p<0.05$ wholebrain cluster correction and overlaid on the patient's own T1 structural scan with the subdural electrode grid superimposed.

\section{Results}

\section{Behavioral results for ECoG and fMRI}

Table 2 contains the behavioral data. For ECoG, go trial RT was fairly quick (mean $=572 \mathrm{~ms}$ ), there was a low number of dis- 
Table 2. Behavioral data

\begin{tabular}{llllllll}
\hline Patient & Expt & $\begin{array}{l}\text { RT (ms) } \\
{[\text { median (SD) }]}\end{array}$ & Go errors (\%) & SSRT (ms) & SSD (ms) & $p_{\text {stop }}$ & $\begin{array}{l}\text { Omission errors (\%) (go-critical/ } \\
\text { go-noncritical/stop-noncritical trials) }\end{array}$ \\
\hline TS007 (day 1) & ECOG & $689(178)$ & 1.17 & 220 & 304 & 66.4 & $0.78 / 5.08 / 8.59$ \\
TS007 (day 2) & ECOG & $699(225)$ & 0.39 & 248 & 288 & 63.3 & $3.12 / 1.17 / 7.81$ \\
TS005 & ECOG & $494(138)$ & 1.39 & 351 & 158 & 45 & $0 / 0.63 / 5.0$ \\
TA341 & ECOG & $512(134)$ & 4.69 & 214 & 255 & 54 & $0.52 / 0 / 8.33$ \\
& FMRI & $538(102)$ & 5.2 & 278 & 358 & 53 & NA \\
TA344 & ECOG & $466(111)$ & 3.32 & 360 & 158 & 24 & $0 / 0.52 / 1.04$ \\
& fMRI & $550(89)$ & 2.6 & 368 & 228 & 49 & NA
\end{tabular}

RT is for go trials (no stop signal); omission errors are percentages of trials on which the subject did not respond (shown separately for the different trial types on which responses needed to be made). Expt, Experiment.
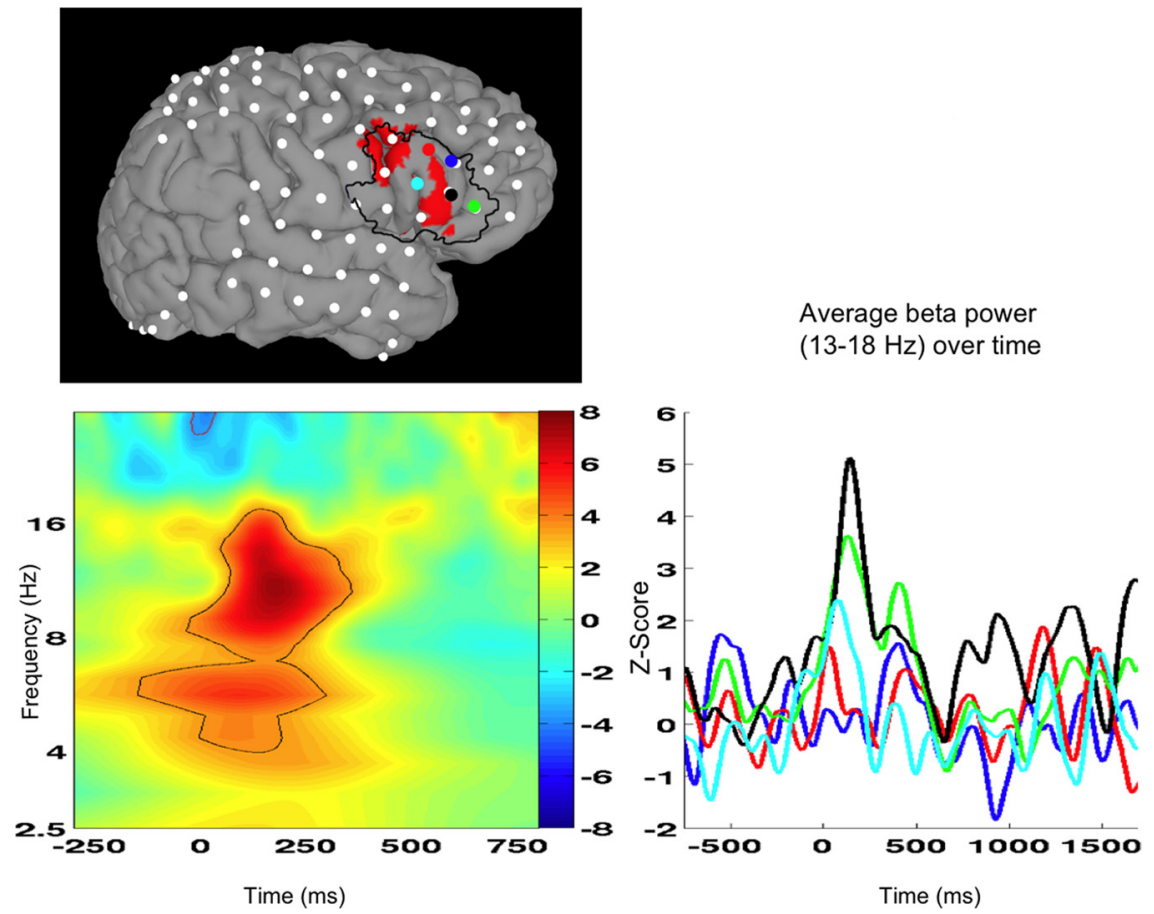

Figure 4. Correspondence between $\mathrm{FMRI}$ and ECOG for patient TA341. Functional MRI activation represents the contrast suc cessful stop - go (red). The time-frequency plot (bottom left) shows ECOG data from one electrode (marked in black on the MRI) for successful stop trials. Zero ms is the time of the stop signal. Right, Beta power over time for this and several other electrodes in the region. The color of each trace corresponds to the color of the electrode shown on the structural MRI. The electrode marked in black was selected for further analysis and had both the closest spatial correspondence to right IFG fMRI activation and also the strongest response ( $z$ score $\sim 6$ ). [Note that the other electrodes in the IFG were excluded because of electrical contamination.]

stop trials compared to go trials $(p<0.05$, whole-brain corrected) (Fig. 4), consistent with prior results in healthy control subjects (Aron and Poldrack, 2006; Aron et al., 2007; Xue et al., 2008). Strikingly, the electrode that was closest to this showed the strongest ECoG result (in terms of $z$ score) for successful stop trials (Fig. 4). The contrast of go-null also activated the right sensorimotor region, as expected ( $p<0.05$, whole-brain corrected) (data not shown).

For TA344, although we obtained fMRI and ECoG for this patient, a portion of the right prefrontal region was determined to be pathological (i.e., showed ictal activity) and was subsequently resected. Therefore, we do not provide right IFG fMRI and ECoG results for this patient. However, we do analyze ECoG M1 data for this patient. For fMRI, the contrast of go-null activated the right sensorimotor region, as expected $(p<0.05$, whole-brain corrected) (data not shown).

\section{Electrode selection}

A key issue was deciding which right IFG electrode within the rather large, probabilistically determined (Eickhoff et al., 2005) anatomical region to analyze in each patient in an unbiased way. For TA341, for

crimination errors on go trials (mean $=2.27 \%$ ), and the patients made relatively few errors of omission on trials for which a response was required (mean for go-critical trials $=0.89 \%$; mean for gononcritical trials $=1.4 \%$; mean for stop noncritical trials $=6.2 \%$ ). With regard to trials on which stopping was required, the mean SSRT was $279 \mathrm{~ms}$, the mean stop-signal delay was $233 \mathrm{~ms}$ and the mean probability of stopping was $50.3 \%$. An anomaly was the low stopping rate $(24 \%)$ in patient TA344. This related to a poor choice of starting stop-signal delay for this ECoG session. Overall, however, it is clear that the ECoG patients performed the task well.

For the two patients with fMRI, the behavioral scores also suggested satisfactory performance and SSRT was roughly consistent with ECoG even though this was a slightly different stopping paradigm $(\mathrm{TA} 341 \mathrm{ECoG}=214 \mathrm{~ms}$, fMRI $=278 \mathrm{~ms}$; TA344 $\mathrm{ECoG}=360 \mathrm{~ms}$, fMRI $=368 \mathrm{~ms}$ ).

\section{Functional MRI results and correspondence with ECoG electrodes}

For patient TA341, there was significantly greater activation in the pars triangularis/opercularis of the right IFG for successful whom we had fMRI data, we used the electrode within the right IFG that corresponded with the maximum peak in BOLD signal for the fMRI contrast of successful stop-go trials (Fig. 4). For TS007 and TS005, where fMRI data were not available, we used the following method. For each right IFG electrode, time-frequency activity was computed for all stop trials (compared to baseline) for the period ranging from the stop signal to $1 \mathrm{~s}$ later. Of these, we selected as the key electrode the one with the strongest response, where this was defined as having the maximum number of points (across time and frequency band) whose absolute value surpassed the $z$ score threshold of $85 \%$ of the maximum $z$ score for all stop trials from all electrodes in the right IFG region for each patient individually. This method takes into account both the strength of the power modulation (in $z$ score) and also how long the modulation lasted, without creating bias toward time or frequency band (supplemental Fig. 1, available at www.jneurosci.org as supplemental material).

The primary motor cortex electrodes were selected based on the anatomically defined hand region (as specified by a neurosurgical expert, N.T.). For TS007 there was only one clear candidate 

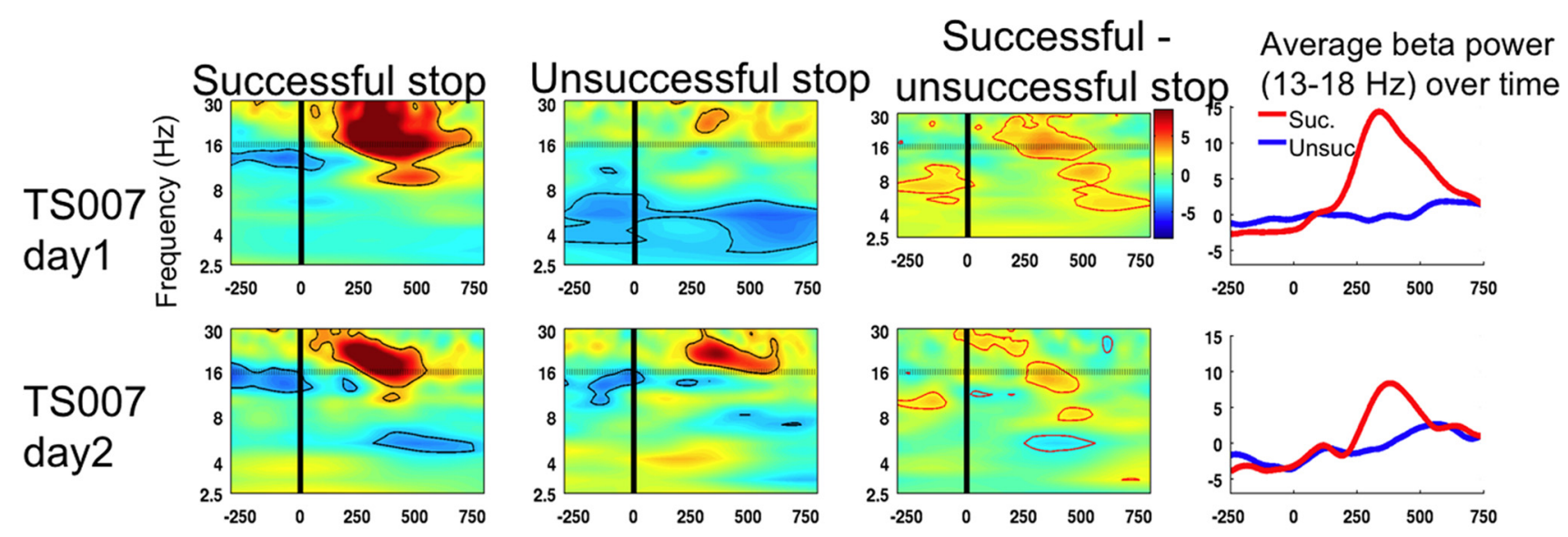

TS005
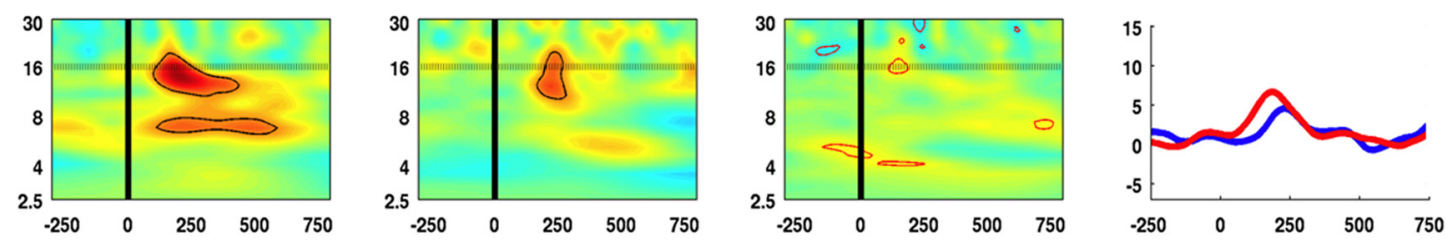

TA341
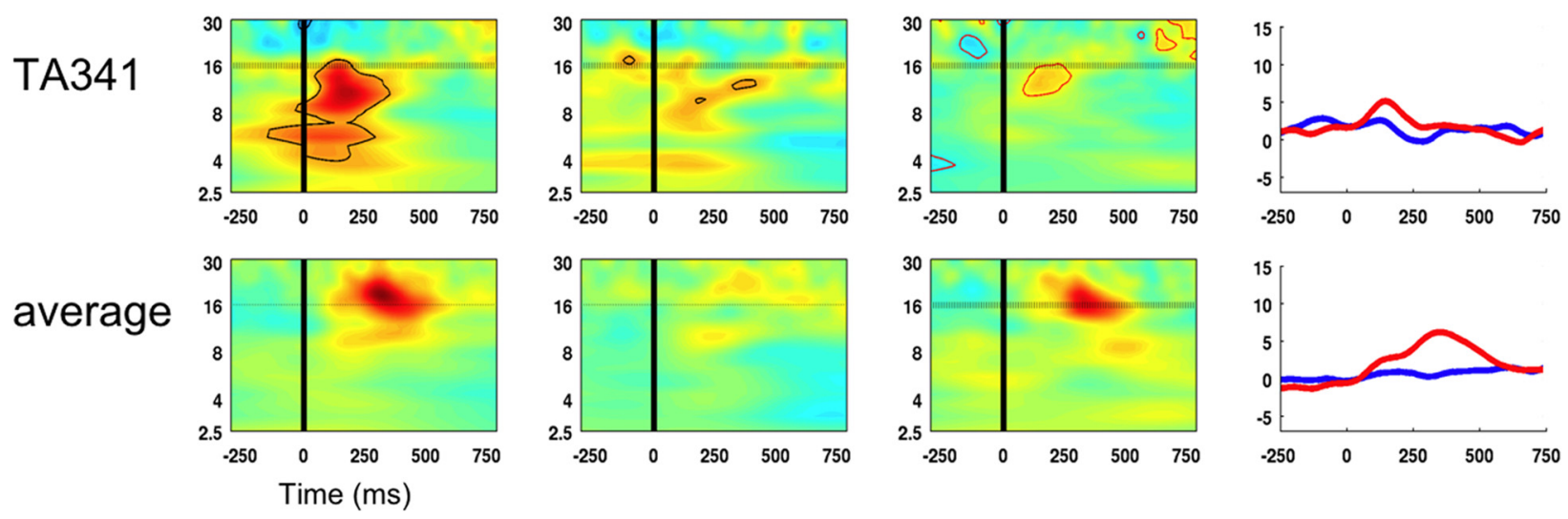

Figure 5. Right IFG results for ECOG. The figure shows a stronger ECoG response for successful versus unsuccessful stop trials. Data are shown for three patients, and for one patient there were $2 \mathrm{~d}$ (sessions). Zero milliseconds is the time of the stop signal, indicated by a thick vertical black line. The first two columns show data from each condition relative to the baseline period as a $z$ score. The third column shows the difference between conditions (relative to one another, not to the baseline), also as a z score. The fourth column shows the same data with power averaged across the beta band (13-18 Hz), plotted for both conditions over time (red line indicates successful stopping; blue line indicates unsuccessful stopping). In all cases there is a beta increase ( $\sim 16 \mathrm{~Hz}$ ) that is larger for successful than for unsuccessful stop trials. The thin black outlines in columns 1 and 2 indicate $p<0.01$, FDR corrected. The thin red outlines shown in the difference column indicate $p<0.05$, uncorrected. The dotted horizontal line marks $16 \mathrm{~Hz}$ for all patients. The bottom row shows the average of $z$ scores across patients, obtained by averaging the average $z$ scores for each patient, down-sampled to $100 \mathrm{~Hz}$ to be comparable across patients.

within the region. For TA341, there was only one electrode in the region which was not contaminated with electrical noise. For TA344, there was no electrode in the region, but we analyzed the closest neighbor.

\section{ECoG results: right IFG}

Having chosen a right IFG electrode for each patient/session (one patient was tested twice) according to the above criteria (except for TA344, who was determined to have ictal activity in this region) we present data from these electrodes for successful and unsuccessful stop trials, and the difference between them, all time locked to the stop signal (Fig. 5). There are a total of four sessions, because TS007 was tested twice over $2 \mathrm{~d}$ - once using the left hand, once with the right hand. We focused on the range of $2.5-30 \mathrm{~Hz}$ to be consistent across all sessions. Within each patient's data, there is significant above-baseline activation at $\sim 13-16 \mathrm{~Hz}$ (beta band) following the stop signal for both successful and unsuccessful stop trials (each comparison $p<0.01$, FDR corrected). Moreover, in all sessions, the power of this $\sim 16$
$\mathrm{Hz}$ activation was larger for successful than unsuccessful stop trials ( $p<0.05$, uncorrected). To evaluate this result further, we used the masking approach (see Materials and Methods). We derived a mask of beta band activity between 0 and $1 \mathrm{~s}$ after the stop signal, from the contrast of all stop trials compared to baseline (FDR corrected, $p<0.01$ ). We examined the $z$ scores at all data points within the mask for the contrast of successful and unsuccessful stop trials. A $\chi^{2}$ test for each patient/session confirmed that the number of data points that surpassed significance ( $p<0.05$, uncorrected) was much greater than expected by chance ( $\chi^{2}$ statistic was $>10,000$ and $p \ll 0.01$ for each session). An important point is that the right frontal response for successful stop trials had some spatial specificity in that the response was stronger within the right IFG region than outside it (supplemental Fig. $1 A-D$, available at www.jneurosci.org as supplemental material).

Notably, the difference between successful and unsuccessful stop trials emerged at $\sim 100$ to $250 \mathrm{~ms}$ after the stop signal for all sessions. This suggests that the right IFG response does not merely relate to auditory stop-signal processing itself because cortical 

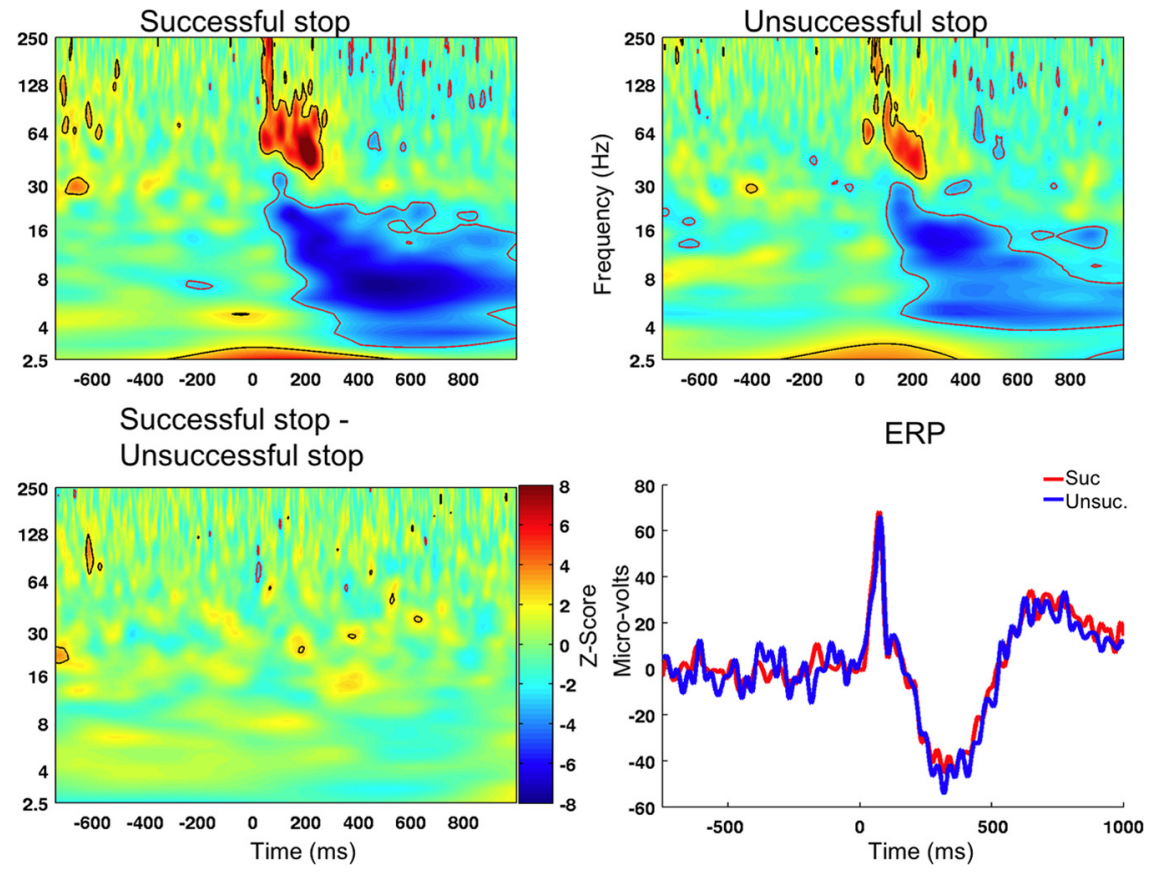

Figure 6. A representative electrode from auditory cortex. This shows a very different pattern of response for successful versus unsuccessful stop trials compared to right IFG. These data are from one electrode for patient, TS007 (for other patients, see supplemental Fig. 2, available at www.jneurosci.org as supplemental material). No substantial, statistically significant differences are present for the contrast of these event types (see difference plot). The event-related potential (low-pass filtered at $40 \mathrm{~Hz}$ ) shows an almost exactly overlapping response between conditions, which begins very shortly after the stop signal ( $<50 \mathrm{~ms})$.

responses to auditory stimuli are detectable in much less than 100 ms (Arezzo et al., 1975; Liegeois-Chauvel et al., 1991). We provide a useful control by analyzing ECoG data from an electrode over auditory cortex in each patient (supplemental Fig. 2, available at www.jneurosci.org as supplemental material). Referring to a representative electrode for patient TS007, day 2 (Fig. 6), note that in auditory cortex, unlike frontal cortex, the response for successful and unsuccessful stop trials was highly similar. Moreover, it was also a much earlier response than observed in prefrontal cortex (occurring at $\sim 50 \mathrm{~ms}$ after the stop signal) consistent with previous findings (Pantev et al., 1991). This suggests that early sensory processing (at least) is identical in both conditions, and thus it is unlikely that the reduced right IFG response in the unsuccessful stop condition relates to differences in stop-signal processing.

Another possible explanation for the difference between successful and unsuccessful stop trials in right IFG electrodes is that, according to the race model (Logan and Cowan, 1984), the go process is slower on successful than on unsuccessful stop trials. This difference in the speed of going could potentially explain the difference in beta frequency observed in the right IFG for successful compared to unsuccessful stop trials. This predicts that slow-go trials will have increased beta frequency power compared to fast-go trials. To examine this possible confound, we performed a median split for each patient to examine slow-go versus fast-go trials and we time locked the ECoG analysis to the arrow onset. Note that we used go-noncritical trials because go-critical trials may include a proactive inhibitory control component that itself activates the right IFG (Jahfari et al., 2009). The result, across several sessions, is not consistent with a greater beta frequency band response on slow-go trials compared to fast ones (supplemental Fig. 3, available at www.jneurosci.org as supplemental material). This suggests that the greater right IFG response on successful versus unsuccessful stop trials does not relate to the speed of going; instead, we expect it relates to stopping.

\section{ECoG results: right $\mathrm{M} 1$}

A downstream target of inhibitory control is M1 (Stinear et al., 2009). Figure 7 shows the ECoG response for a right M1 electrode for the three patients in which the task was performed with the left hand (TS007 d 1, TA341, and TA344). For each patient, and each event type, time locked to the go signal (on critical trials), there was a significant broadband alpha/beta power decrease relative to prestimulus baseline ( $p<0.01$, FDR corrected). This power decrease, or "desynchronization," denotes a pattern that lacks regular periodicity in a particular frequency range relative to baseline. Alpha/beta desynchronization has been previously observed in M1 for movement generation (Crone et al., 1998; Pfurtscheller et al., 2003; Alegre et al., 2006). Consistent with this we found significant alpha/beta desynchronization on unsuccessful stop trials $(p<0.01$, FDR corrected). Moreover, we also observed significant alpha/beta desynchronization for successful stop trials $p<0.01$, FDR corrected), but it was diminished. This difference between successful and unsuccessful trials was significant ( $p<0.05$, uncorrected). Again, to evaluate this difference further, we took the masking approach (see Materials and Methods). We derived a mask of alpha and beta band activity $(8-30 \mathrm{~Hz})$ between 0 and $1 \mathrm{~s}$ after the go signal, from the contrast of all stop trials compared to baseline (FDR corrected, $p<0.01$ ). We examined the $z$ scores at all data points within the mask for the contrast of unsuccessful and successful stop trials. A $\chi^{2}$ test for each patient confirmed that the number of data points that surpassed significance $(p<0.05$, uncorrected) was much greater than expected by chance ( $\chi^{2}$ statistic was $>10,000$ and $p \ll 0.01$ for each patient). We note that the smaller desynchronization for successful versus unsuccessful stop trials probably does not merely reflect the fact that there was movement for unsuccessful stop trials while there was none for successful stop trials. Instead, we expect it relates to the stopping of an initiated response. We note that the successful stop trials showed a desynchronization relative to a nonmovement baseline. We argue that this reflects the initiated movement, which is subsequently stopped.

\section{Discussion}

Much evidence implicates the right IFG, along with the dorsomedial frontal cortex, the basal ganglia, and the primary motor cortex, in stop-signal and go-no-go response inhibition (Schall et al., 2002; Rubia et al., 2003; Garavan et al., 2006; Li et al., 2006, 2008; Aron et al., 2007; Isoda and Hikosaka, 2007, 2008; Mostofsky and Simmonds, 2008; Chambers et al., 2009; Stinear et al., 2009). Here we sought to better characterize the functional role of the right IFG and primary motor cortex in behavioral stopping with ECoG.

For the right IFG, there was a significant above-baseline response for successful stop trials and also for unsuccessful stop trials in the beta frequency band $(\sim 16 \mathrm{~Hz})$ in the $100-500 \mathrm{~ms}$ 


\section{Unsuccessful stop Successful stop}

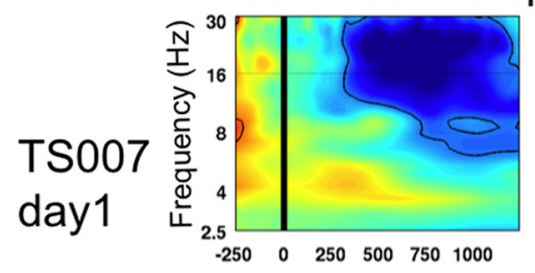

TA344

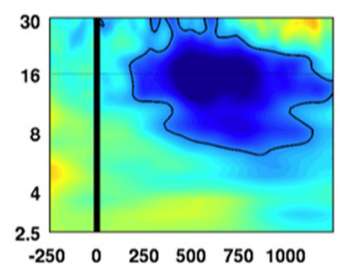

TA341
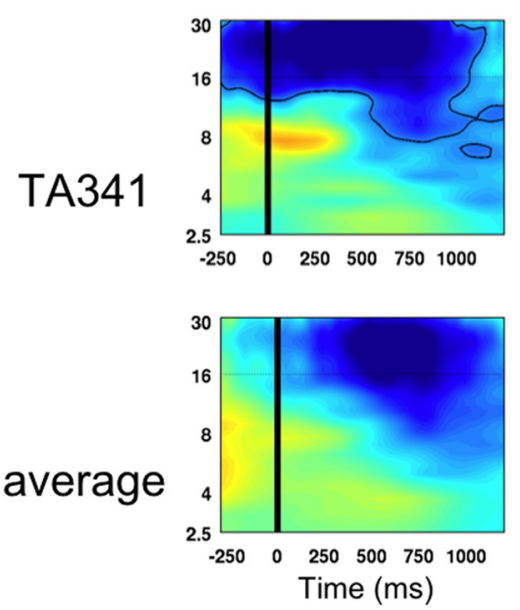
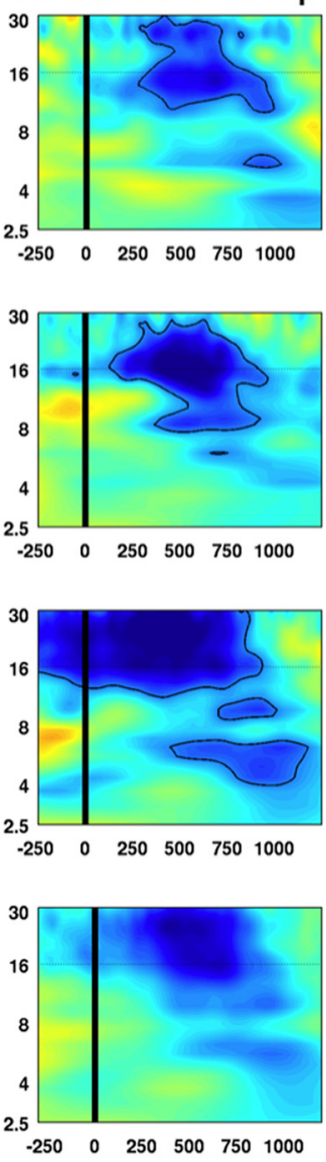

Unsuccessful -

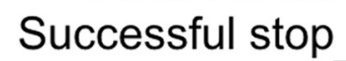

Average alpha/beta power (8-30 Hz) over time
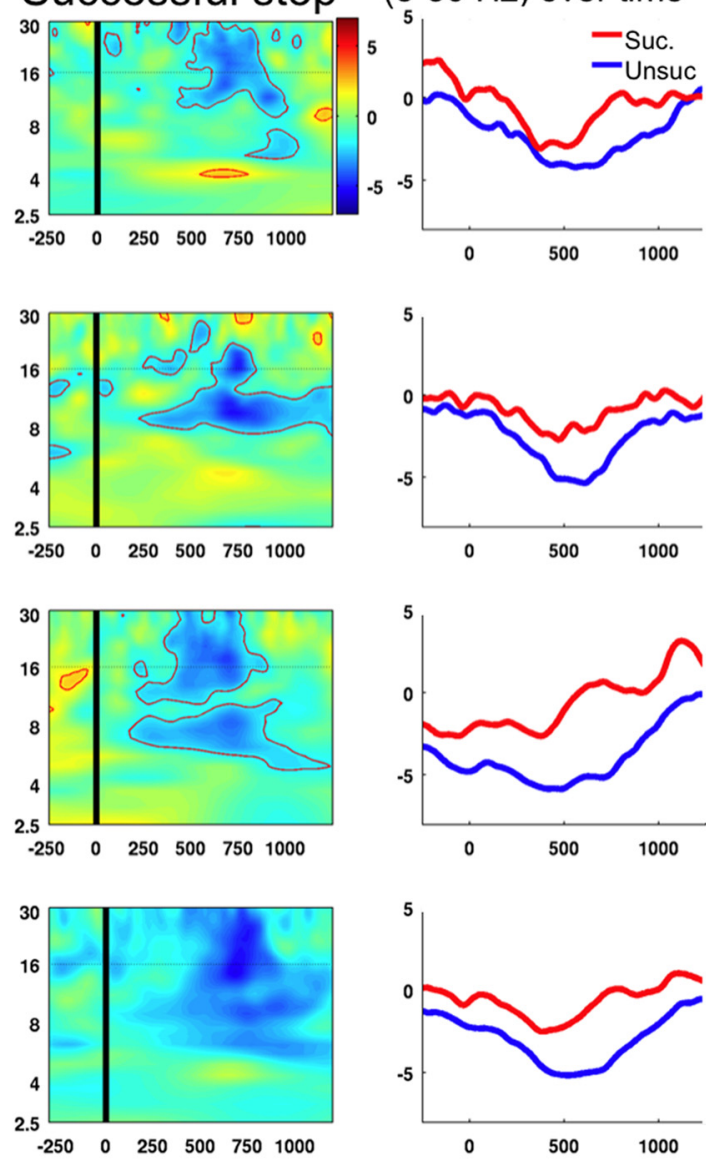

Figure 7. Right primary motor cortex results for ECOG. There is a different ECoG response for successful versus unsuccessful stop trials. Zero milliseconds is the time of the go signal, indicated by a thick vertical black line. The first two columns show data from each condition relative to the baseline period (intertrial interval) as a $z$ score. The third column shows the difference between conditions (relative to one another, not to the baseline), also as a z score. The fourth column shows average alpha/beta power $(8-30 \mathrm{~Hz})$ over time. As for Figure 5 , thin black outlines indicate $p<$ 0.01 , FDR corrected; thin red outlines in the difference column indicate $p<0.05$, uncorrected; dotted horizontal line marks $16 \mathrm{~Hz}$ for all patients. The bottom row shows the average of $z$ scores across patients. Responses were made with the left hand.

range after the stop signal, and the response on successful stop trials was stronger than unsuccessful stop trials. These findings provide novel evidence regarding the functional role of the right IFG in behavioral stopping. First, the right IFG response on stop trials began $100-250 \mathrm{~ms}$ after the stop signal. This is roughly consistent with the N200 event-related potential observed in many scalp EEG studies of stopping (Kok et al., 2004; Schmajuk et al., 2006; Liotti et al., 2007; Dimoska and Johnstone, 2008). The increase we observed between 100 and $250 \mathrm{~ms}$ for successful and unsuccessful trials (and the difference) is unlikely related to mere auditory processing of the stop signal because this latency is much too late to reflect a cortical response to the tone (which may occur within $13 \mathrm{~ms}$ in auditory cortex and not much later in frontal cortex) (Arezzo et al., 1975; Liegeois-Chauvel et al., 1991). Indeed, in the auditory cortex of our patients there were power modulations very soon after the stop signal that did not differ for successful versus unsuccessful stop trials. Thus, the right IFG response is probably not related to mere stop-signal detection.

Another important aspect of the stopping-related increase in right IFG power was that it was most prominent in the beta frequency band. This is striking because other studies show motor-related effects in the beta frequency range when recording from the subthalamic nucleus (Kühn et al., 2004, 2005; Kloster- mann et al., 2007). In particular, the study by Kühn et al. (2004) reported beta frequency power increases on no-go trials relative to go trials. This suggests that an increase in beta power could relate to behavioral response inhibition in the subthalamic nucleus, as it does in the right IFG. Other evidence shows that the subthalamic nucleus plays a role in stop-signal and other kinds of response inhibition (Aron and Poldrack, 2006; van den Wildenberg et al., 2006; Chamberlain et al., 2008; Eagle et al., 2008; Isoda and Hikosaka, 2008; Ray et al., 2009) and human tractography suggests it is connected with the right IFG via a "hyperdirect" tract (Aron et al., 2007). Furthermore, coupling between the subthalamic nucleus and other frontal regions involved in motor networks occurs in the beta band (Lalo et al., 2008). It is thus possible that the beta frequency response of the right IFG reflects functional communication with the subthalamic nucleus within a structurally connected network that supports inhibitory control. This is a testable hypothesis, for example in patients undergoing simultaneous cortical and subcortical recording during task performance.

These findings in the right IFG converge with other data (for review, see Chambers et al., 2009) to point to a stopping-related function implemented in this region. While being able to stop successfully presumably requires an inhibitory control function, 
it must also require working memory and forms of attention (Corbetta and Shulman, 2002; Aron and Poldrack, 2005; Hampshire et al., 2007; Mostofsky and Simmonds, 2008). We cannot rule out the possibility that it is these attentional/mnemonic processes which relate to the right IFG response observed here rather than inhibitory control per se. However, it is likely that a putative right IFG inhibitory function would need to be closely coupled with attentional/mnemonic processes, perhaps implemented in the same region. Future research is needed to examine this question.

On a technical note, prior research reports alpha/beta power increases that follow decreases, and this is taken to reflect an "idle" or suppressed state (Pfurtscheller et al., 1996). Yet here the right IFG beta power increase was not a "rebound" of a previous power decrease. Rather, it followed the onset of the tone at 100$250 \mathrm{~ms}$, with activity before the tone similar to the baseline intertrial interval. Thus, the well known beta patterns associated with movement may not hold for areas outside the sensorimotor cortex such as the prefrontal cortex. Instead, changes in beta power outside the sensorimotor cortex could be interpreted as signaling an "active-akinetic" process rather then a lack of movement (Brown and Williams, 2005).

A puzzling feature of our data (in common with scalp EEG findings) is that the ECoG response was greater for successful than unsuccessful stop trials, whereas some functional MRI studies have only found reliable activation of the right IFG region for the contrast of successful stopping with go trials (Aron and Poldrack, 2006; Aron et al., 2007; Xue et al., 2008; but see Rubia et al., 2003; Li et al., 2006). Indeed a well powered fMRI metaanalysis from 101 subjects showed that the contrast of successful and unsuccessful stop trials did not activate the right IFG region (E. Congdon, G. Xue, A. R. Aron, and R. A. Poldrack, unpublished observations). There are several possible reasons for this discrepancy between ECoG and fMRI data. First, according to the race model, whether a patient stops is determined by the speed of the go and the stop process (Logan and Cowan, 1984). In healthy participants the success of stopping may largely relate to variability in going, rather than stopping, and this may explain why both successful and unsuccessful stop trials activate the right IFG similarly in some studies. In our patients, however, stopping may not always be triggered (e.g., due to fatigue); hence, a difference would emerge between successful and unsuccessful stop trials. Second, there are differences in what is measured by fMRI and ECoG. While both methods are sensitive to EPSPs, the BOLD response is more related to higher-frequency bands than to lower ones such as the beta band response observed here (Logothetis et al., 2001; Mukamel et al., 2005). Third, differences in activity for successful versus unsuccessful stop trials may occur for too short a time period to be detected with fMRI, whereas the difference between successful stop and go trials is more substantial (so it can be observed with fMRI). Finally, ECoG (but not fMRI) is sensitive to synchrony between and within regions. Thus, the difference between successful and unsuccessful stop trials may not be a difference in the firing rate of right IFG neurons, nor their input or local activity, but rather the degree of synchrony within the right IFG or between the right IFG and the basal ganglia. This could produce a different ECoG response for successful and unsuccessful stop trials but no difference for the BOLD signal (Ray et al., 2008).

Turning next to M1, there was a low-frequency power decrease (desynchronization) for unsuccessful stop trials. This is consistent with prior reports of alpha/beta desynchronization for M1 associated with movement (Crone et al., 1998; Pfurtscheller et al., 2003). This is a validation of our data collection and analysis techniques and of the assumption that nonpathologic brain regions of epilepsy patients may serve as good models. Importantly, there was less desynchronization for successful compared to unsuccessful stop trials. Notably, TMS studies have shown that successful stop trials are associated with more GABA-mediated inhibition in M1 than go trials (Sohn et al., 2002; Coxon et al., 2006; van den Wildenberg et al., 2009). We thus speculate that the diminished desynchronization on successful stop trials may be due to increased GABAergic inhibition. Indeed, GABA agonists (benzodiazepines) have been shown to modulate beta activity in M1 (Jensen et al., 2005). This study thus contributes potentially useful information about the relationship between frequency band, behavioral control, and neuromodulatory tone in primary motor cortex.

This study had the following limitations. (1) One of the patients we studied (TA 344) underwent a subsequent right prefrontal resection; therefore, right ECoG data are not presented for this person (although M1 data are). (2) The sampling rate was different across patients and, in some cases, lower than desirable (overall limiting examination of higher frequencies). Indeed, our not reporting high-frequency responses should not be taken to show that such ranges are not important for prefrontal function and stopping (Canolty et al., 2006). (3) fMRI was not acquired in all patients; thus, the observed correspondence between ECoG and BOLD signal in right IFG is preliminary.

In summary, we used the high spatiotemporal resolution afforded by ECoG to clarify the functional role of the right IFG in stop-signal response inhibition. There was a significant abovebaseline response for each of successful and unsuccessful stop trials in the beta frequency band $(\sim 16 \mathrm{~Hz})$ in the range $100-500$ $\mathrm{ms}$ after the stop signal, with the response being stronger on successful than unsuccessful stop trials. The finding that right IFG activity begins $\sim 100-250 \mathrm{~ms}$ after the stop signal suggests that it is not merely related to the detection of the signal, and the finding that it ends by $\sim 500 \mathrm{~ms}$ suggests that it is not merely related to processing the success (or not) of stopping. Instead it likely reflects a function that is key to the behavioral stopping itself, as suggested by converging evidence from loss-of-function studies (Chambers et al., 2009). The observation that the right IFG response occurred in the beta frequency band provides novel information in support of the hypothesis that the right IFG is part of a network which includes the subthalamic nucleus of the basal ganglia (Aron et al., 2007). We also examined the downstream effects of behavioral stopping in primary motor cortex. Making a movement on unsuccessful stop trials was associated with a strong below-baseline desynchronization, whereas successfully stopping an initiated movement was associated with a less strong desynchronization. We interpret this reduction in desynchronization on successful stop trials in the context of other studies as an increase in GABA-mediated inhibition in primary cortex (Sohn et al., 2002; Jensen et al., 2005; Coxon et al., 2006; van den Wildenberg et al., 2009). Together, these findings provide convergent evidence for a fronto/basal ganglia/primary cortical motor network for stopping initiated responses, and they point to the importance of inter-regional binding by synchronized neuronal activity in the beta frequency range for likely functional connectivity within this network.

\section{References}

Alegre M, Imirizaldu L, Valencia M, Iriarte J, Arcocha J, Artieda J (2006) Alpha and beta changes in cortical oscillatory activity in a go/no go randomly-delayed-response choice reaction time paradigm. Clin Neurophysiol 117:16-25. 
Arezzo J, Pickoff A, Vaughan HG Jr (1975) The sources and intracerebral distribution of auditory evoked potentials in the alert rhesus monkey. Brain Res 90:57-73.

Aron AR, Poldrack RA (2005) The cognitive neuroscience of response inhibition: relevance for genetic research in attention-deficit/hyperactivity disorder. Biol Psychiatry 57:1285-1292.

Aron AR, Poldrack RA (2006) Cortical and subcortical contributions to Stop signal response inhibition: role of the subthalamic nucleus. J Neurosci 26:2424-2433.

Aron AR, Fletcher PC, Bullmore ET, Sahakian BJ, Robbins TW (2003) Stopsignal inhibition disrupted by damage to right inferior frontal gyrus in humans. Nat Neurosci 6:115-116.

Aron AR, Behrens TE, Smith S, Frank MJ, Poldrack RA (2007) Triangulating a cognitive control network using diffusion-weighted magnetic resonance imaging (MRI) and functional MRI. J Neurosci 27:3743-3752.

Brown P, Williams D (2005) Basal ganglia local field potential activity: character and functional significance in the human. Clin Neurophysiol 116:2510-2519.

Canolty RT, Edwards E, Dalal SS, Soltani M, Nagarajan SS, Kirsch HE, Berger MS, Barbaro NM, Knight RT (2006) High gamma power is phaselocked to theta oscillations in human neocortex. Science 313:1626-1628.

Canolty RT, Soltani M, Dalal SS, Edwards E, Dronkers NF, Nagarajan SS, Kirsch HE, Barbaro NM, Knight RT (2007) Spatiotemporal dynamics of word processing in the human brain. Front Neurosci 1:185-196.

Chamberlain SR, Hampshire A, Müller U, Rubia K, Del Campo N, Craig K, Regenthal R, Suckling J, Roiser JP, Grant JE, Bullmore ET, Robbins TW, Sahakian BJ (2009) Atomoxetine modulates right inferior frontal activation during inhibitory control: a pharmacological functional magnetic resonance imaging study. Biol Psychiatry 65:550-555.

Chambers CD, Bellgrove MA, Stokes MG, Henderson TR, Garavan H, Robertson IH, Morris AP, Mattingley JB (2006) Executive "brake failure" following deactivation of human frontal lobe. J Cogn Neurosci 18:444-455.

Chambers CD, Bellgrove MA, Gould IC, English T, Garavan H, McNaught E, Kamke M, Mattingley JB (2007) Dissociable mechanisms of cognitive control in prefrontal and premotor cortex. J Neurophysiol 98:3638-3647.

Chambers CD, Garavan H, Bellgrove MA (2009) Insights into the neural basis of response inhibition from cognitive and clinical neuroscience. Neurosci Biobehav Rev 33:631-646.

Chen CY, Muggleton NG, Tzeng OJ, Hung DL, Juan CH (2009) Control of prepotent responses by the superior medial frontal cortex. Neuroimage 44:537-545.

Chevrier AD, Noseworthy MD, Schachar R (2007) Dissociation of response inhibition and performance monitoring in the stop signal task using event-related fMRI. Hum Brain Mapp 28:1347-1358.

Corbetta M, Shulman GL (2002) Control of goal-directed and stimulusdriven attention in the brain. Nat Rev Neurosci 3:201-215.

Cox RW (1996) AFNI: software for analysis and visualization of functional magnetic resonance neuroimages. Comput Biomed Res 29:162-173.

Coxon JP, Stinear CM, Byblow WD (2006) Intracortical inhibition during volitional inhibition of prepared action. J Neurophysiol 95:3371-3383.

Crone NE, Miglioretti DL, Gordon B, Lesser RP (1998) Functional mapping of human sensorimotor cortex with electrocorticographic spectral analysis. II. Event-related synchronization in the gamma band. Brain 121:2301-2315.

Dale AM, Fischl B, Sereno MI (1999) Cortical surface-based analysis. I. Segmentation and surface reconstruction. Neuroimage 9:179-194.

Dimoska A, Johnstone SJ (2008) Effects of varying stop-signal probability on ERPs in the stop-signal task: do they reflect variations in inhibitory processing or simply novelty effects? Biol Psychol 77:324-336.

Eagle DM, Baunez C, Hutcheson DM, Lehmann O, Shah AP, Robbins TW (2008) Stop-signal reaction-time task performance: role of prefrontal cortex and subthalamic nucleus. Cereb Cortex 18:178-188.

Eickhoff SB, Stephan KE, Mohlberg H, Grefkes C, Fink GR, Amunts K, Zilles K (2005) A new SPM toolbox for combining probabilistic cytoarchitectonic maps and functional imaging data. Neuroimage 25:1325-1335.

Floden D, Stuss DT (2006) Inhibitory Control is Slowed in Patients with Right Superior Medial Frontal Damage. J Cogn Neurosci 18:1843-1849.

Fries P (2005) A mechanism for cognitive dynamics: neuronal communication through neuronal coherence. Trends Cogn Sci 9:474-480.

Garavan H, Hester R, Murphy K, Fassbender C, Kelly C (2006) Individual differences in the functional neuroanatomy of inhibitory control. Brain Res 1105:130-142.

Hampshire A, Duncan J, Owen AM (2007) Selective tuning of the blood oxygenation level-dependent response during simple target detection dissociates human frontoparietal subregions. J Neurosci 27:6219-6223.

Isoda M, Hikosaka O (2007) Switching from automatic to controlled action by monkey medial frontal cortex. Nat Neurosci 10:240-248.

Isoda M, Hikosaka O (2008) Role for subthalamic nucleus neurons in switching from automatic to controlled eye movement. J Neurosci 28:7209-7218.

Jahfari S, Stinear CM, Claffey M, Verbruggen F, Aron AR (2009) Responding with restraint: what are the neurocognitive mechanisms? J Cogn Neurosci, in press.

Jensen O, Goel P, Kopell N, Pohja M, Hari R, Ermentrout B (2005) On the human sensorimotor-cortex beta rhythm: sources and modeling. Neuroimage 26:347-355.

Klostermann F, Nikulin VV, Kühn AA, Marzinzik F, Wahl M, Pogosyan A, Kupsch A, Schneider GH, Brown P, Curio G (2007) Task-related differential dynamics of EEG alpha- and beta-band synchronization in corticobasal motor structures. Eur J Neurosci 25:1604-1615.

Kok A, Ramautar JR, De Ruiter MB, Band GP, Ridderinkhof KR (2004) ERP components associated with successful and unsuccessful stopping in a stop-signal task. Psychophysiology 41:9-20.

Konishi S, Nakajima K, Uchida I, Kikyo H, Kameyama M, Miyashita Y (1999) Common inhibitory mechanism in human inferior prefrontal cortex revealed by event-related fMRI. Brain 122:981-991.

Kühn AA, Williams D, Kupsch A, Limousin P, Hariz M, Schneider GH, Yarrow K, Brown P (2004) Event-related beta desynchronization in human subthalamic nucleus correlates with motor performance. Brain 127:735-746.

Kühn AA, Trottenberg T, Kivi A, Kupsch A, Schneider GH, Brown P (2005) The relationship between local field potential and neuronal discharge in the subthalamic nucleus of patients with Parkinson's disease. Exp Neurol 194:212-220

Lalo E, Thobois S, Sharott A, Polo G, Mertens P, Pogosyan A, Brown P (2008) Patterns of bidirectional communication between cortex and basal ganglia during movement in patients with Parkinson disease. J Neurosci 28:3008-3016.

Li CS, Huang C, Constable RT, Sinha R (2006) Imaging response inhibition in a stop-signal task: neural correlates independent of signal monitoring and post-response processing. J Neurosci 26:186-192.

Li CS, Yan P, Sinha R, Lee TW (2008) Subcortical processes of motor response inhibition during a stop signal task. Neuroimage 41:1352-1363.

Liegeois-Chauvel C, Musolino A, Chauvel P (1991) Localization of the primary auditory area in man. Brain 114:139-151.

Liotti M, Pliszka SR, Perez R 3rd, Luus B, Glahn D, Semrud-Clikeman M (2007) Electrophysiological correlates of response inhibition in children and adolescents with ADHD: influence of gender, age, and previous treatment history. Psychophysiology 44:936-948.

Logan G (1994) On the ability to inhibit thought and action: a users' guide to the stop signal paradigm. In: Inhibitory processes in attention, memory and language (Dagenbach D, Carr TH, eds). San Diego: Academic.

Logan GD, Cowan WB (1984) On the ability to inhibit thought and action: a theory of an act of control. Psychol Rev 91:295-327.

Logothetis NK, Pauls J, Augath M, Trinath T, Oeltermann A (2001) Neurophysiological investigation of the basis of the fMRI signal. Nature 412:150-157.

Mars RB, Coles MG, Hulstijn W, Toni I (2008) Delay-related cerebral activity and motor preparation. Cortex 44:507-520.

Mostofsky SH, Simmonds DJ (2008) Response inhibition and response selection: two sides of the same coin. J Cogn Neurosci 20:751-761.

Mukamel R, Gelbard H, Arieli A, Hasson U, Fried I, Malach R (2005) Coupling between neuronal firing, field potentials, and FMRI in human auditory cortex. Science 309:951-954.

Pantev C, Makeig S, Hoke M, Galambos R, Hampson S, Gallen C (1991) Human auditory evoked gamma-band magnetic fields. Proc Natl Acad Sci U S A 88:8996-9000.

Pfurtscheller G, Stancák A Jr, Neuper C (1996) Post-movement beta synchronization. A correlate of an idling motor area? Electroencephalogr Clin Neurophysiol 98:281-293.

Pfurtscheller G, Graimann B, Huggins JE, Levine SP, Schuh LA (2003) Spatiotemporal patterns of beta desynchronization and gamma syn- 
chronization in corticographic data during self-paced movement. Clin Neurophysiol 114:1226-1236.

Ray NJ, Jenkinson N, Brittain J, Holland P, Joint C, Nandi D, Bain GP, Yousif N, Green A, Stein JS, Aziz TZ (2009) The role of the subthalamic nucleus in response inhibition: evidence from deep brain stimulation for Parkinson's disease. Neuropsychologia 28:11526-11536.

Ray S, Crone NE, Niebur E, Franaszczuk PJ, Hsiao SS (2008) Neural correlates of high-gamma oscillations $(60-200 \mathrm{~Hz})$ in macaque local field potentials and their potential implications in electrocorticography. J Neurosci 28:11526-11536.

Rubia K, Smith AB, Brammer MJ, Taylor E (2003) Right inferior prefrontal cortex mediates response inhibition while mesial prefrontal cortex is responsible for error detection. Neuroimage 20:351-358.

Schall JD, Stuphorn V, Brown JW (2002) Monitoring and control of action by the frontal lobes. Neuron 36:309-322.

Schmajuk M, Liotti M, Busse L, Woldorff MG (2006) Electrophysiological activity underlying inhibitory control processes in normal adults. Neuropsychologia 44:384-395.

Sohn YH, Wiltz K, Hallett M (2002) Effect of volitional inhibition on cortical inhibitory mechanisms. J Neurophysiol 88:333-338.

Stinear CM, Coxon JP, Byblow WD (2009) Primary motor cortex and movement prevention: where Stop meets Go. Neurosci Biobehav Rev 33:662-673.

Studholme C, Novotny E, Zubal IG, Duncan JS (2001) Estimating tissue deformation between functional images induced by intracranial electrode implantation using anatomical MRI. Neuroimage 13:561-576.

van den Wildenberg WP, van Boxtel GJ, van der Molen MW, Bosch DA, Speelman JD, Brunia CH (2006) Stimulation of the subthalamic region facilitates the selection and inhibition of motor responses in Parkinson's disease. J Cogn Neurosci 18:626-636.

van den Wildenberg WP, Burle B, Vidal F, van der Molen MW, Ridderinkhof KR, Hasbroucq T (2009) Mechanisms and dynamics of cortical motor inhibition in the stop-signal paradigm: a TMS study. J Cogn Neurosci, in press.

Verbruggen F, Logan GD (2009) Models of response inhibition in the stopsignal and stop-change paradigms. Neurosci Biobehav Rev 33:647-661.

Xue G, Aron AR, Poldrack RA (2008) Common neural substrates for inhibition of spoken and manual responses. Cereb Cortex 18:1923-1932.

Zandbelt B, Van Buuren M, Gladwin TE, Hoogendam R, Kahn S, Vink M (2008) Brain regions involved in response inhibition are also activated during anticipation of inhibition. Soc Neurosci Abstr 34:672.19. 